\title{
A HYBRID ROUTING PROTOCOL IN WIRELESS MESH NETWORKS
}

\author{
Nikolaos Peppas and Damla Turgut \\ School of EECS \\ University of Central Florida \\ Orlando, Florida 32816 \\ Email: \{peppas,turgut\}@eecs.ucf.edu
}

\begin{abstract}
Wireless mesh networks (WMN) is a new promising wireless technology which uses already available hardware and software components. In this paper, we propose a hybrid routing algorithm for military applications. More specifically, a specialized scenario consisting of a network of flying Unmanned Aerial Vehicles (UAVs) executing reconnaissance missions is investigated. Simulation experiments are conducted to evaluate the performance of our algorithm in terms of the routing overhead, latency, and packet delivery ratio with respect to varying number of nodes and node density. Three classes of node mobility: low, medium, and high are considered in the simulation study. The results showed that the latency tends to increase as the network grows larger. All the metrics revealed sensitivity in high mobility conditions.
\end{abstract}

\section{INTRODUCTION}

As technology evolves, wireless networks are becoming more and more widespread since they play an essential role in providing better services in every day life. A new key technology called wireless mesh networks (WMN) is estimated to contribute significantly in the next generation of wireless computing [1], [2]. Mesh networks consist of two types of nodes: mesh routers and mesh clients. The routers form the backbone of such a network and they can perform conventional operations similar to any wireless router. What differentiates a mesh router is its capability of having multiple wireless network interfaces operating on multiple channels which can maximize the throughput and the overall network performance. Power consumption is less of an issue since most of the routing decisions are made by the mesh routers. Mesh routers are also responsible for making the different radio technologies (WiFi, WiMax, etc.) compatible with each other. On the other hand, there are the mesh clients which can be any devices such as laptops, PDAs, pocket PCs, IP phones, and so on. A mesh client usually has one network interface and it is much simpler that a mesh router. Clients can easily connect to the network by getting automatically detected and they disconnect without affecting the network reliability and performance.

There are three types of mesh architectures [2]. First, there is the infrastructure or backbone architecture, where wireless mesh routers provide a backbone topology which is used by the clients to connect and operate. Communication between the routers is accomplished through wireless protocols such as 802.11. One or more routers can function as a gateway for providing internet connection to the entire network. The second type is the client architecture which is a peer-to-peer way of communication where data is transmitted directly from one node to another. If the sender does not have the destination within its transmission range, the data is transmitted through other intermediate clients. In other words, client architecture is very similar to multi-hop ad hoc network topology. The specialized scenario investigated in this paper corresponds to this type of architecture, with the UAVs being the client nodes. Finally, there is the hybrid mesh architecture which combines characteristics of both of the previous two types of setups. In other words, there is the backbone section of the network which supports part of the clients; however there are also clients which communicate among themselves in a peer-to-peer mode.

Regardless of the way a mesh network is deployed, various applications will be enhanced by the realization of WMN, such as home and enterprise networking, building automation and networking in unreachable urban areas. The main advantages of this new technology are the ease and low cost of the deployment. Most of the components required are already available in the networking community consisting both hardware components as well as established and tested software protocols.

This paper proposes a hybrid routing algorithm for a specialized scenario of a mesh network consisting of moving UAVs possibly to be used by the Air Force to investigate new grounds. It combines characteristics of both proactive and reactive routing protocols currently used in ad hoc networks. The protocol is evaluated based on routing overhead, latency, and packet delivery ratio performance metrics. A stand alone simulator, based on Java, is used to carry out the simulation study since well-known network 
simulators such as ns-2 [3] and GloMoSim [4] are not feasible options for wireless mesh networks. Among the different research challenges that mesh networking faces, routing is one of the most crucial to the overall performance of the network.

The reminder of the paper is as follows. Section II describes the literature work in the areas of mesh networking and routing protocols. Section III presents our proposed hybrid mesh routing protocol in detail. The simulation environment, metrics, and results are explained in Section IV. Section V concludes the paper.

\section{RELATED WORK}

The ease of deployment and the compatibility with other wireless technologies are some of the few reasons why mesh networking has a high potential of overtaking the market in the near future. WMN nodes can communicate both with other clients, forming a client architecture, and with mesh routers. They are also flexible in terms of mobility and their ability to carry multiple radios with multiple frequencies which provides them with great potential to achieve high throughput performance.

[5] is an effort made by an MIT team to provide an area with internet access through a wireless mesh network backbone. The project is widely known as Roofnet. More specifically 37 nodes spread in an area of $4 \mathrm{~km}^{2}$ in the city of Manchester, MA in an unplanned manner. Various performance parameters of the topology are evaluated such as link throughput relation with node density as well as antenna placement strategies. Regardless of the lack of planning, the randomly deployed mesh network offers an average throughput of $627 \mathrm{kbits} / \mathrm{sec}$ even though the average route has 3 hops. Such a throughput can be considered more than enough for every day usage and despite the long routes it performs much better than the potential performance of a single-hop network. Using omnidirectional antennas at each node is the key to the high throughput measurements.

A new metric, called Expected Transmission Count (ETX), used by multi-hop routing protocols for path selection is presented in [6]. It predicts the total number of transmissions (including retransmissions) until it is delivered to its destination. In order to compute its value, it uses the per link loss ratio in both directions of each wireless link. It was tested in a 29 node testbed and proved to have excellent performance even as the network grows larger and the links become longer.

Based on this idea, a new metric, called Estimated Transmission Time (ETT) was introduced in [7]. This metric not only uses ETX mentioned above, but also considers the bandwidth of each link. All the ETT link weights are combined together resulting in a Weighted Cumulative ETT (WCETT) that describes each path. This metric is the core of the multi-radio link-quality source routing protocol. In contrast with ETX [6], the simulations run on the 23 node testbed show that this metric performs well in multi-channel environments.

The ETT metric is also used in another routing protocol technique which appears in [8]. In order to utilize the diversity of the communication channels in a more efficient fashion, this protocol proposed the opportunistic usage of multiple paths simultaneously. While most multi-path protocols use the first identified path for transmission, they start using the other available paths in case of failure. In [8], the multiple packets are forwarded through all the identified routes at the same time. This method was proven to increase the throughput significantly since more data was transmitted through multiple paths.

\section{PROPOSED MESH ROUTING PROTOCOL}

\section{A. System design}

The military is the main application of the proposed protocol in this paper. The network should guarantee the ability to rapidly deploy a force to any location in the world and instantly communicate using high data rates. This communication can provide live feeds of audio and video from the individual teams executing engagements for training or live combat to their base stations, which can in turn function as routers/gateways to the internet. Thus, the proposed routing algorithm is intended to provide the network with such capabilities and is evaluated through a scenario which considers a set of unmanned aerial vehicles (UAVs) above a certain terrain. The downstream data is usually larger than the upstream data since the ultimate goal for the flying reconnaissance planes is to send information to the base stations which could serve as routers/gateways to the internet. However, there are also communication messages transmitted between the UAVs which help them coordinate their terrain identification process. There is a variety of the UAVs operating at various speeds, altitudes and paths, as depicted in Figure 1.

- Class A: High-altitude, high-speed UAVs are taking reconnaissance data at the altitude of 40,000 feet and speed of around $800 \mathrm{~km} / \mathrm{h}$.

- Class B: Medium-altitude, medium-speed UAVs are patrolling certain areas at the altitude of 20,000 feet at a speed of around $200-300 \mathrm{~km} / \mathrm{h}$.

- Class C: Low-altitude, low-speed UAVs which are hovering or moving with slow speed around a relatively static area. Their altitude can be $0-1,000$ feet and their speed of $0-100 \mathrm{~km} / \mathrm{h}$. The ground nodes which can be either slow moving battle tanks or base stations 
serving as gateways for data processing and internet connection also belong to this class.

Let us now consider a typical scenario of the system. More specifically, there are several UAVs deployed and controlled from a base station. A class A reconnaissance airplane identifies a potential point of interest. Initially, it scans the specific area and transmit the information to any base station. However, due to its high speed it can only observe the target for a limited time and with minimal level of detail. Therefore, it can communicate with a lower class airplane and pass the observation task to have a better image of the area. This process can also happen the other way around. A lower class node can ask a higher class airplane to get a wider view of a certain area. As a result, we have two types of data to transmit: communication data which coordinates the assigned tasks to the UAVs as well as images or video of the scanned target. Class $\mathrm{C}$ planes usually just serve the role of intermediate nodes to the task of data delivery to the ground stations.

\section{B. The protocol description}

The proposed routing protocol uses attributes and characteristics of the current ad hoc routing protocols since the situation is similar. In ad hoc networks, we have two main types of routing protocols: proactive and reactive. In our case, the decision was to use both routing methods taking into consideration the class of each node.

More specifically, class A nodes use reactive while class $\mathrm{B}$ and $\mathrm{C}$ nodes follow proactive routing protocols as can be seen in Figure 1. Reactive routing causes flooding which usually affects the performance of the network negatively. However, since the nodes are moving at a very high speed any table-driven protocol could have a high number of invalid entries resulting in high packet loss ratio. That is why reactive routing is preferred at this level. In addition, class A nodes are not the busiest ones since the highest load is in the two bottom classes of nodes. So, it is obvious that in the upper node class there is a tradeoff between the performance and the data delivery assurance.

The reason the class $B$ and $C$ nodes use the proactive routing is that the mobility is not as high; however, the packet overhead is expected to be relatively high due to the periodic table updates. This would be a waste of bandwidth in cases where the nodes are not used frequently. However, class $\mathrm{B}$ and $\mathrm{C}$ nodes are busy transmitting their own data as well as forwarding other nodes' data. As a result, having extra overhead becomes valuable since it would contribute to the assurance of the successful packet delivery.

In order to better understand the structure of the logic through which the protocol works, a very straightforward pseudocode is provided in Algorithm 1.

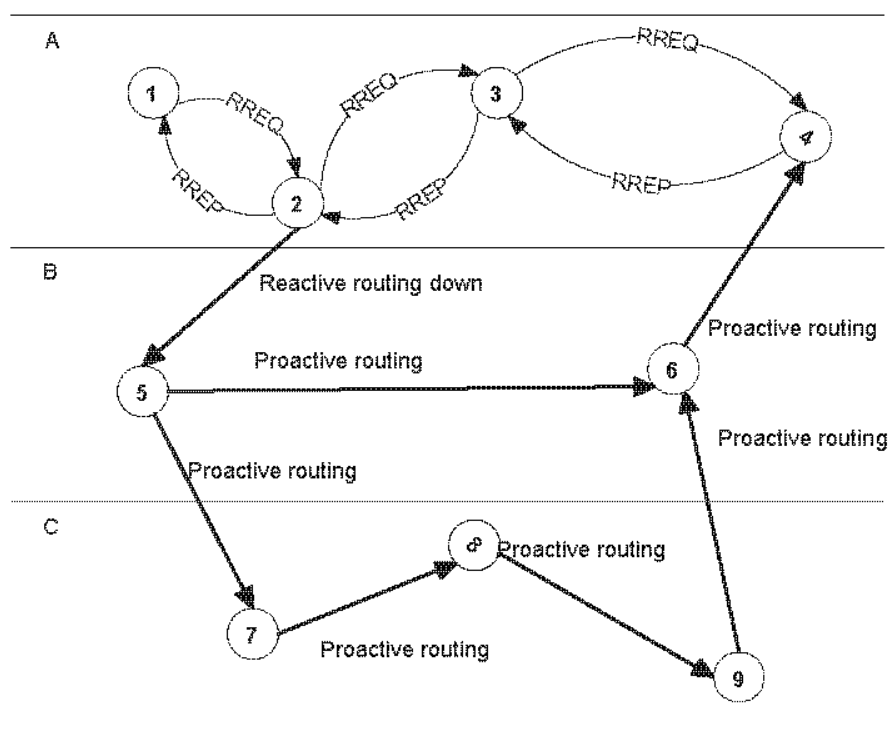

Fig. 1. Protocol usage according to node class.

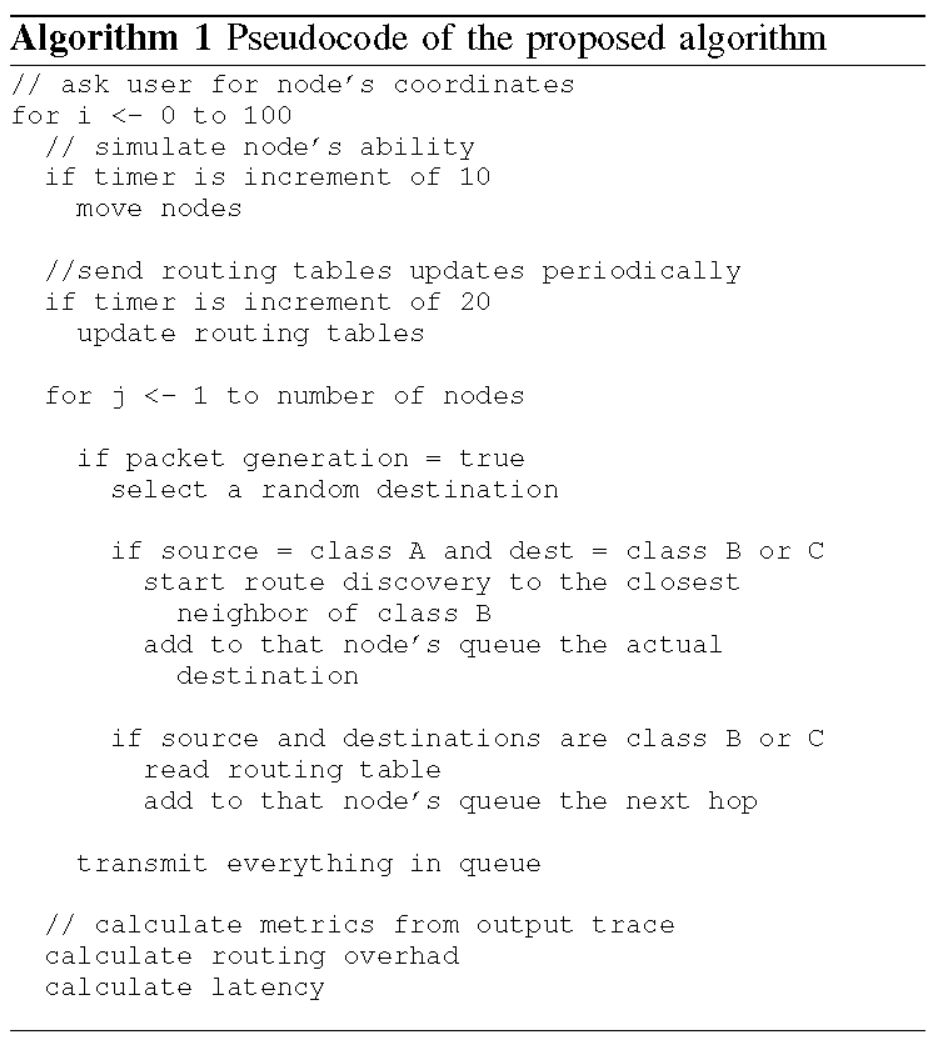

\section{SIMULATION STUDY}

This section provides detailed presentation of the simulation environment, metrics, and the results. The algorithm simulates the movement of UAVs and collects routing data. Our simulation study concentrates on the network layer and collects data about the behavior and performance of the proposed protocol. We assume the existence of an ideal collision-free MAC layer. 


\section{A. Simulation environment}

The simulator itself is a stand alone program written in Java2. The environment used to develop the source code is Eclipse [9]. The mechanism generates a random number of events among the nodes and it logs every transmission in an output trace file. At the end, this file is processed in order to extract the protocol metrics. The input of the algorithm is the total number of nodes participating in the simulation and their initial coordinates. The altitude the nodes are allowed to fly is assumed to be between $0-400$ units $(0-40,000 \mathrm{ft})$. If a node is placed within $0-132$ units of altitude then it is class $\mathrm{C}$ (lower class). In the same manner, nodes between 133-264 are class B and the upper class nodes are placed from 265-400. Nodes are considered within transmission range of each other if their distance is less than 60 units. In general, there are more nodes flying at lower altitudes than at higher altitudes. In our simulation, the proportionality of the nodes in a given class is consistent and complies with the relation count $(\mathrm{A})<\operatorname{count}(\mathrm{B})<\operatorname{count}(\mathrm{C})$.

The nodes are following a mobility pattern in which class A nodes move the fastest, class $\mathrm{B}$ nodes move slower than class $\mathrm{A}$ but faster than class $\mathrm{C}$ and the bottom class nodes are the slowest of all, if not stationary. A summary of the simulation parameters can be seen in Table I.

\section{B. Simulation metrics}

We evaluated the performance of our protocol by the following metrics.

- \% Routing overhead: The routing overhead is the packets used for routing table updates as well as control packets from the reactive part of the protocol (RREQ, RREP). It is calculated using the following formula:

$$
\begin{aligned}
& \% \text { Overhead }= \\
& \quad\left[\frac{\text { Overhead packets }}{\text { Overhead packets }+ \text { Data packets }}\right] \times 100
\end{aligned}
$$

- Latency: The time that takes a packet to arrive at the destination. It is measured in time units with a time unit being the amount of time for a packet to reach a one hop destination. All links are considered to have equal cost.

- Packet delivery ratio: It is the ratio of the number of packets successfully delivered over the total packets transmitted in the simulation:

$$
\begin{aligned}
& \text { Packet Delivery Ratio }= \\
& {\left[\frac{\text { delivered packets }}{\text { delivered packets }+ \text { lost packets }}\right] \times 100}
\end{aligned}
$$

We study the variation of these three metrics in function of the following simulation parameters:

- Number of nodes: The amount of nodes participating in the simulation, ranging from 3 to 21 . Regardless of the number of nodes used in the simulation, the UAVs are positioned, in such a way that they provide a node density of about 1 node in every $10 \mathrm{~km}^{2}$.

- Node density: The network topology is usually affecting the network performance. Node density, which describes the deployment of the nodes, is the number of nodes encountered in every $10 \mathrm{~km}^{2}$. The values range from 0.6 to 1.4 nodes per $10 \mathrm{~km}^{2}$. While evaluating the importance of node density, the number of nodes in the topology is maintained at 12 nodes.

- Node mobility: Mobility is a crucial factor that affects the mesh networks. We have chosen low, medium, and high mobility patterns. In every position change each node shifts its $\mathrm{x}$ and $\mathrm{y}$ coordinates by a distance within specified range in Table II. Through this method random variations in the speed of the node provide more realistic conditions for the simulation.

TABLE II

A SUMMARY OF THE MOBILITY PATTERNS USED.

\begin{tabular}{ll}
\hline Low mobility pattern \\
\hline Class A node position shift range & $120-150 \mathrm{~m}$ \\
Class B node position shift range & $30-90 \mathrm{~m}$ \\
Class $C$ node position shift range & $0-30 \mathrm{~m}$ \\
\hline Medium mobility pattern & \\
\hline Class A node position shift range & $150-240 \mathrm{~m}$ \\
Class B node position shift range & $60-120 \mathrm{~m}$ \\
Class $C$ node position shift range & $0-30 \mathrm{~m}$ \\
\hline High mobility pattern & \\
\hline Class $A$ node position shift range & $180-300 \mathrm{~m}$ \\
Class B node position shift range & $60-180 \mathrm{~m}$ \\
Class $C$ node position shift range & $0-30 \mathrm{~m}$ \\
\hline
\end{tabular}

\section{Simulation results}

1) \% Routing overhead: In this experiment, we measure the routing overhead in function of the number of nodes. The measurements for the three different levels of mobility are shown in Figure 2. We observe that for a small network size, the overhead starts with $45 \%$, but decreases and stays around $30 \%$ as the network gets larger. Generally, $30 \%$ is a relatively high overhead for routing algorithms. In our case it is caused by the frequent refreshes of the routing tables, as well as the relatively short data transmission sequences. We note that there is little variation on the routing overhead with the increase of the number of nodes. The node mobility does not seem to affect much the routing overhead.

Next, we compared the overhead with respect to the changing node density and how this relation is affected by the different mobility patterns. As mentioned earlier density is described by the number of nodes in every $10 \mathrm{~km}^{2}$. The larger the number is, the denser the topology. Figure 3 shows the routing overhead in relation to the node density. 
TABLE I

SIMULATION PARAMETERS.

\begin{tabular}{ll}
\hline Simulation Parameters & Value \\
\hline Simulation time & 1000 time units \\
Number of nodes & $3-21$ \\
Node transmission range & $24,000 \mathrm{ft}$ \\
Routing tables update interval & 20 time units \\
Node position change interval & 10 time units \\
Node class quantity relation $(A: B: C)$ & count $(\mathrm{A})<\operatorname{count}(\mathrm{B})<\operatorname{count}(\mathrm{C})$ \\
Node potential altitude & $0-40,000 \mathrm{ft}$ \\
\hline
\end{tabular}

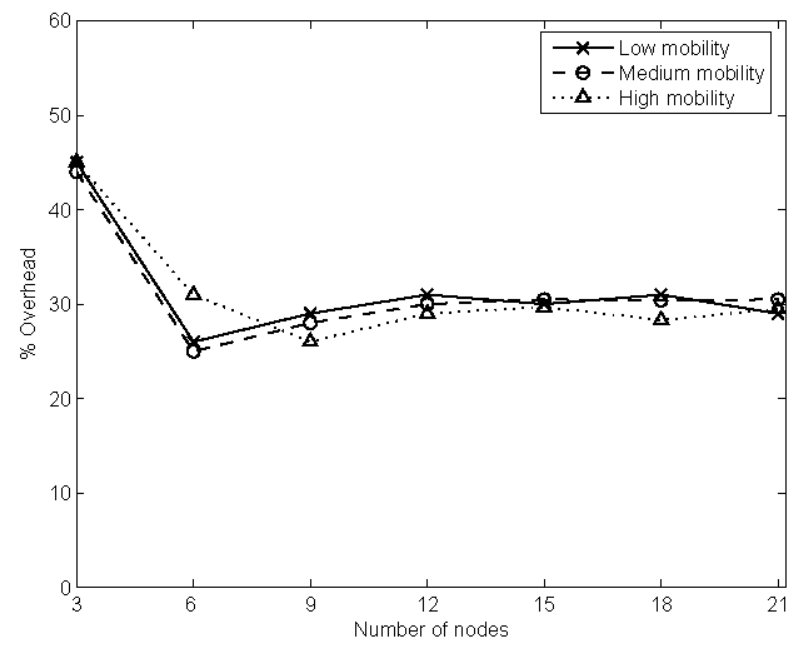

Fig. 2. Average routing overhead vs. number of nodes for the three classes of mobility.

The resulting values are in the range of $25-33 \%$ with an average value of $30 \%$. This shows that routing overhead is not affected by the node density. Similarly, the mobility of the nodes does not alter the relation between packet overhead and node density. The nodes broadcast the routing updates in periodic intervals regardless of the density of the network or the speed of the moving UAVs. However, the updates would get affected with the increase in the quantity of the nodes in each node class. For example, placing several class A nodes and less class B and C would definitely result in variation of the overhead. However, this is not the case since all simulations followed the same deployment guidelines which give a standard node quantity relation of count $(\mathrm{A})<\operatorname{count}(\mathrm{B})<\operatorname{count}(\mathrm{C})$ for classes $\mathrm{A}$, $\mathrm{B}$ and $\mathrm{C}$ respectively.

Overall, it appears that routing overhead has a steadily high value because of the routing table updates and it neither degrades nor improves by varying the number of nodes or the node density. The only parameter that could affect the routing overhead is the time period that the nodes send table updates to their neighbors as well as the

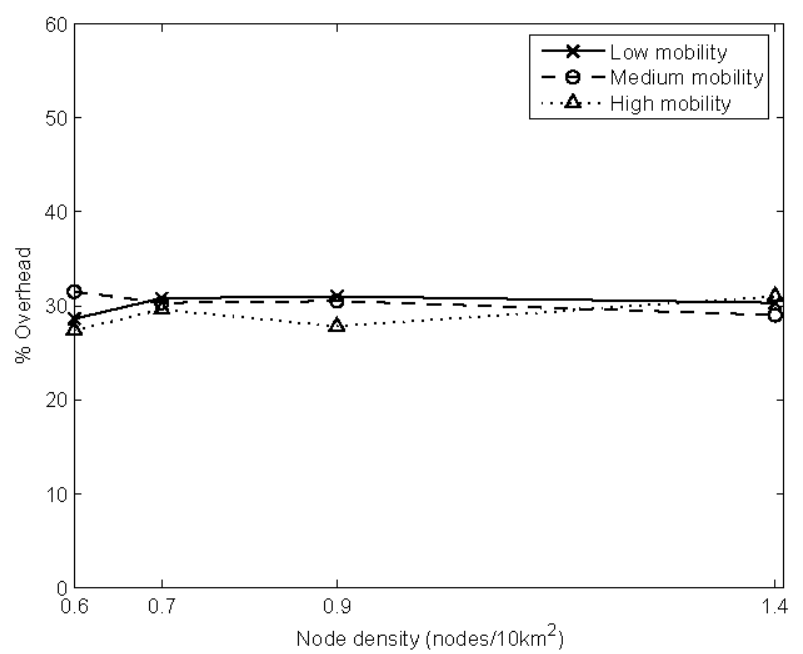

Fig. 3. Average routing overhead vs. node density for the three classes of mobility (number of nodes $=12$ ).

proportionality of nodes within the topology. Since neither of these were changed during the simulations, it is expected for the overhead to remain almost unchanged.

2) Latency: Figure 4 illustrates the latency in relation to the number of nodes. We can draw a conclusion that the protocol shows high potential towards scalability. On the average, it takes 1.2-2.3 time units for a packet delivery. Since a time unit is the time for a packet transmission from one node to another (1-hop), the latency can be interpreted as the number of hops. Usually, the more hops it takes for a packet delivery, the greater the latency is. A slight increase is noticed as more nodes added into the mesh network. This can be explained by the fact that with additional number of nodes the potential distance from any source to any destination gets larger and a longer path would be followed. We also observed that the average latency is higher for high mobility scenarios. The high mobility can cause the distances between source and destination pairs to get larger, in turn taking longer time to deliver the packets. This can also mean that additional hops may be needed to reach the destination node.

Next, the latency as a function of node density is exam- 


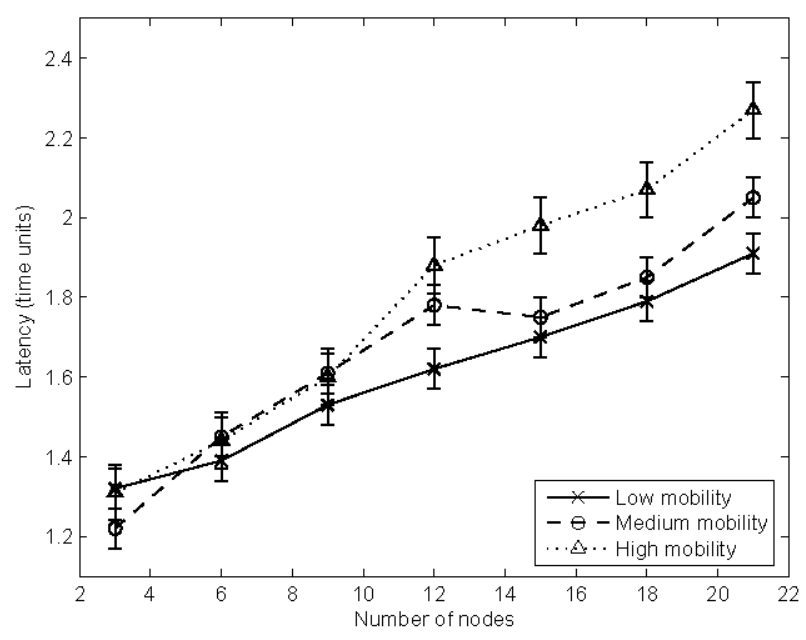

Fig. 4. Average latency vs. number of nodes for the three classes of mobility.

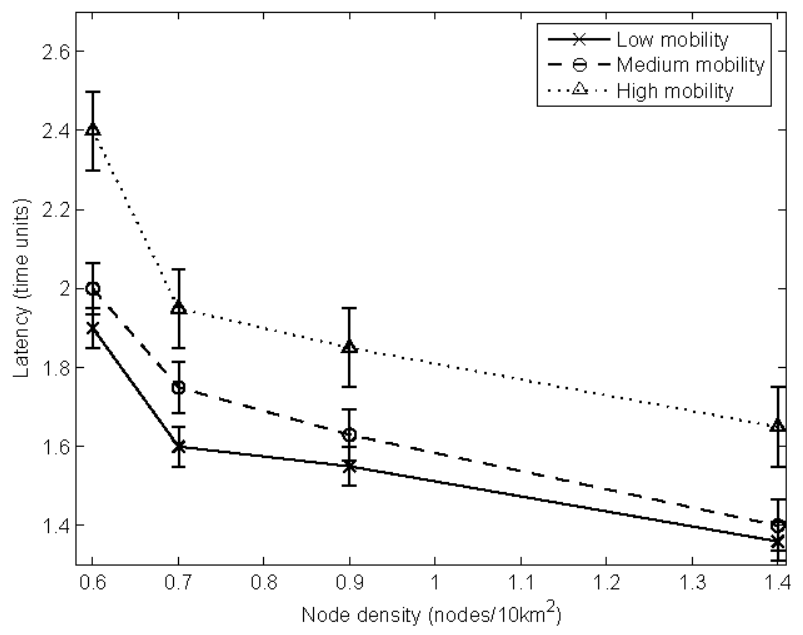

Fig. 5. Average latency vs. node density for the three classes of mobility (number of nodes $=12$ ).

ined for scenarios with the three different levels of mobility in Figure 5. Latency is affected by the node density. While the node density decreases as nodes move away from each other, distances between source and destination pairs become longer creating the need for extra hops in the routing path. The conditions get worse if along with the addition of more nodes, the mobility is also increased. As the distances get longer, it takes more time to deliver the packets causing the latency to escalate.

In summary, it appears that latency is affected by the size of the network and this relation is in turn affected by the mobility pattern followed by the nodes. There is a tendency for the latency to increase as more nodes are added or the existing nodes start to move faster.

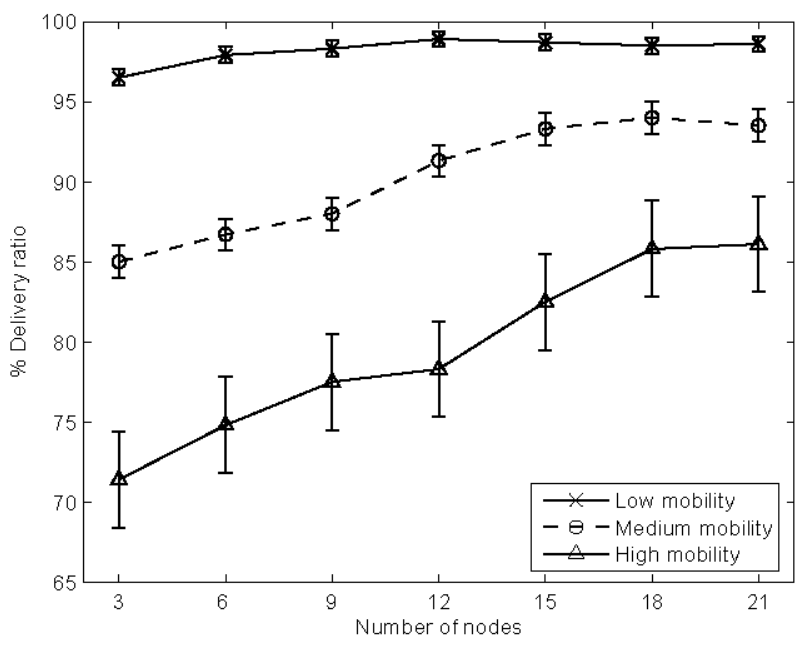

Fig. 6. Average packet delivery ratio vs. number of nodes for the three classes of mobility.

3) Packet delivery ratio: The packet delivery ratio as a function of the number of nodes for the three levels of mobility is considered in Figure 6. If the mobility is at moderate level, one can conclude that for a large network in terms of number of nodes, the delivery ratio may approach to above $90 \%$. Since collisions are not considered here, the packet loss can happen either when there are no neighbor nodes in the node's transmission range or the routing table becomes stale by the time of the packet transmission. Thus, as the network becomes larger the probability of finding a node to forward the packets is higher. Class A nodes with the highest mobility have a higher chance of losing the packets; however, their traffic load is much lower compared to the class $\mathrm{B}$ or $\mathrm{C}$ nodes.

Next, we study the packet delivery ratio as a function of node density for the three classes of mobility in Figure 7. We make note of two trends. First, the packet delivery ratio increases proportionally with the node density due to more available number of nodes within the nodes' transmission range. Second, we observe that the delivery ratio is consistently higher for low node mobility scenario since the routing tables stay fresh longer periods of time.

We can conclude that reasonably high packet delivery ratio is obtained throughout the experiments. We observe that the mesh network is slightly sensitive to node density and highly sensitive to node mobility.

\section{CONCLUSIONS}

We proposed a hybrid routing algorithm for military applications in wireless mesh networks. A specific scenario consisting of high speed unmanned air vehicles (UAVs) was considered. High flying nodes use reactive while low flying 


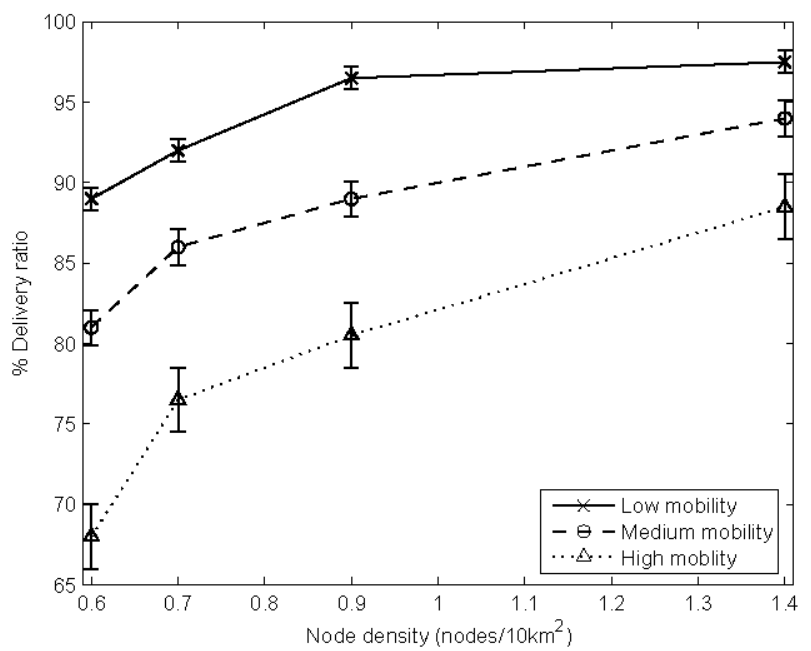

Fig. 7. Average packet delivery ratio vs. node density for the three classes of mobility (number of nodes $=12$ ).

nodes use proactive routing.

Simulation experiments are conducted to evaluate the performance of the proposed protocol in terms of routing overhead, latency, and packet delivery ratio with varying number of nodes, node density, and node mobility. Ideal MAC protocol with no packet collisions is assumed.
Most of the metrics collected reveal positive indications about the performance of the protocol. Latency might be an issue that could cause scalability problems if the number of nodes and mobility get excessive. The simulations showed that there is a tendency for the latency to increase not only when more nodes enter the network but also when mobility is increased.

\section{REFERENCES}

[1] S. Mahmud, S. Khan, S. Khan, and H. Al-Raweshidy, "A comparison of manets and wmns: commercial feasibility of community wireless networks and manets," in Proceedings of the First International Conference on Access Networks (AcessNets'06), 2006, pp. 18-24.

[2] I. Akyildiz and X. Wang, "A Survey on Wireless Mesh Networks," IEEE Communications Magazine, vol. 43, no. 9, pp. 23-30, September 2005 .

[3] "The Network Simulator (ns-2)," http:/www.isi.edu/nsnam/ns.

[4] "Global Mobile Information Systems Simulation Library(glomosim)," http://pcl.cs.ucla.edu/projects/glomosim.

[5] J. Bicket, D. Aguayo, S. Biswas, and R. Morris, "Architecture and Evaluation of an Unplanned 802.11b Mesh Network," in Proceedings of MOBICOM, August 2005, pp. 31-42.

[6] D. D. Couto, D. Aguayo, J. Bicket, and R. Morris, "A HighThroughput Path Metric for Multi-hop Wireless Routing," Wireleless Networking, vol. 11, no. 4, pp. 419-434, 2005.

[7] R. Draves, J. Padhye, and B. Zill, "Routing in Multi-radio, Multihop Wireless Mesh Networks," in Proceedings of MOBICOM, 2004, pp. 114-128.

[8] I. Sheriff and E. Belding-Royer, "Multipath Selection in Multi-radio Mesh Networks," in Broadnets, October 2006.

[9] "Eclipse," http://www.eclipse.org. 